\title{
Effect of Chitosan, Aluminum Oxide and Silver Nanoparticles on Antibacterial, Deodorizing and Moisture Absorption Properties of Nonwoven Polyester Fabrics for Use in Medical Textiles
}

\author{
Mohammad Khajeh Mehrizi \\ (PhD) \\ Department of Textile \\ Engineering, Yazd University, \\ Yazd, Iran \\ Hasan Mashroteh (PhD) \\ Department of Textile \\ Engineering, Yazd University, \\ Yazd, Iran
}

Narges Nabizadeh Moghadam Noghabi (MSc)

Department of Textile

Engineering, Yazd University, Yazd, Iran

Corresponding author:

Mohammad Khajeh Mehrizi

Tel: +983531232591

Email: mkhajeh@yazd.ac.ir

Address: Department of Textile Engineering, Yazd University, Yazd, Iran

\begin{abstract}
Background and Objective: Hospital clothing and textiles have a great role in providing medical services to patients. Hospital services would not be possible without using these textiles. The aim of this study was to prepare non-woven polyester for use in medical textiles.

Methods: In this study, most important expected properties of hospital clothing were created on polyester fabrics via various stages of finishing using chemicals such as calcium chloride, sodium alginate, chitosan and nanoparticles of silver nitrate and aluminum oxide. Antibacterial (activity against Staphylococcus aureus), deodorizing and wicking properties of samples were evaluated using nano-biomaterials via standard tests.

Results: The water absorption rate of nonwoven samples that were finished with chitosan significantly improved. The results also showed favorable level of antimicrobial activity caused by silver nitrate and chitosan. In addition, deodorizing activity was observed in all samples. However, silver nitrate-finished samples had higher deodorizing activity. The capillary action of all samples reduced after completion of all finishing procedures.

Conclusion: The results show that the use of chitosan and alginate along with nanoparticles of silver nitrate and aluminum oxide can be effective in achieving the desired properties of medical textiles.
\end{abstract}

Keywords: Medical textiles, antibacterial substances, chitosan, silver nitrate.

Received : 14 Feb 2015

Revised: 20 Apr 2015

Accepted: 23 Apr 2015 


\section{INTRODUCTION}

Microorganisms are the main cause of infectious diseases. Two different aspects can be considered in antimicrobial protection generated by chemical finishing: textile consumer protection against odor-producing microorganisms (hygienic finish) and textile protection against damage caused by fungi, mold or degrading microorganisms. Under ideal conditions, an antibacterial agent should be able to kill or control a wide range of bacteria using different mechanisms. Silver and its compounds are among the most suitable inorganic antibacterial substances. It is a non-toxic natural mineral metal, capable of destroying a wide range of bacteria and fungi. This ability increases when the particles are in nanometer-size range (1). Due to high surface area to volume ratio, silver nanoparticles have stronger antimicrobial activity than a majority of metals. Studies have demonstrated that even its nanomolar concentrations are effective against bacteria (2). Silver nanoparticles are unable to distinguish between beneficial and harmful bacteria and kill all bacteria in their environment, which is harmful to both humans and the environment. Chitosan is another substance with antimicrobial properties. Chitin and chitosan are natural amino polysaccharides with multi-purpose structure that are widely used in medicine, drug and gene delivery. Their main characteristics are high biocompatibility, acceptable biodegradability with low toxicity, and antibacterial and anti-allergy activity (1). After cellulose, chitin is the second most abundant organic matter in nature (3). Chitosan nanofibers along with silver nanoparticles have been used in electrospun dressings for infected wounds, which have had satisfactory results. This woven product has been used for production of filters, masks, medical supplies, etc. (4). Studies also show that nanosilver alone or in combination with chitosan, prevents the growth of parasites in living tissues (5-8). Moisture transmission through textiles has a great impact on thermophysiological comfort of the human body. Moisture remains on the body in liquid and vapor form. Accordingly, it is necessary to wick sweat out of the clothes to create thermal equilibrium in the human body. Due to their significant surface area, textiles can absorb and retain various gases or volatile substances containing $9 \mathrm{ml}$ of normal saline. from the surrounding environment. Desorption of malodorous materials in vicinity of textiles with high adsorption power can be problematic. Malodor is considered as a serious problem in textiles that have long packaging, shipping and storage time. Bahari et al. investigated use of alginate on polyester warp knitted fabric and evaluated the stability of the formed alginate film on the fabric. Their results showed that applying calcium chloride on fabrics prior to alginate padding causes stronger bonding and increases the stability of alginate (9). Parikh et al. also studied the antimicrobial properties of silver on cotton fabrics and its impact on faster wound healing process (10). However, since cotton is a natural fiber and may become combined with the wound in long-term, polyester fibers were used in this study. On the other hand, Slevam et al. investigated the antimicrobial properties of different substances such as zinc oxide, titanium oxide and silver nanoparticles on cotton fabric, and associated the optimum conditions for antimicrobial properties with finishing with silver nanoparticles (11). The present study tried to increase water-absorbing capacity of non-woven polyester fabric by finishing it with sodium alginate. In addition, the impact of using silver nitrate on the antimicrobial property was studied. The effects of chitosan, sodium alginate and nano-silver nitrate on samples were compared. Finally, nano-aluminum oxide was used for its deodorizing property. The variables used in this study included temperature and type of nano and biomaterials.

\section{MATERIALS AND METHODS}

Needle-punched nonwoven polyester fabric with different weights $(85,140,190$ and $240 \mathrm{~g} / \mathrm{m}^{2}$ ) was used in this study. In addition, chitosan with medium molecular weight and $80 \%$ acetylation (Sigma-Aldrich Co.), silver nitrate nanoparticles with mean size of $20 \mathrm{~nm}$ (Nanoscale Science Research Center, US) and aluminum oxide nanoparticles with mean size of $80 \mathrm{~nm}$ (Neutrino Co.) were used. Moreover, substances such as sodium alginate, calcium chloride, sodium hydroxide and industrial acetic acid were used in this study. Laboratory foulard was used for padding solutions on the samples. SDL-mini dryer-steamer was used for 
drying and fixing the coated (finished) samples. Autoclave was used for sterilizing the samples. An incubator (Tajhiz Azma Co.) was used to provide suitable conditions for bacterial growth at $37{ }^{\circ} \mathrm{C}$ for 24 hours. Transitional spectrophotometer (Spectronic 700) was used for measuring the absorbance of Staphylococcus aureus.

Testing method: First, samples were washed with non-ionic detergent $(2 \mathrm{~g} / \mathrm{l}$ with 1:50 L: R) for 15 minutes and then fixed at $120{ }^{\circ} \mathrm{C}$. In the second phase, samples were pad-coated with calcium chloride solution $(2.5 \mathrm{~g} / \mathrm{l})$ and then dried at $120{ }^{\circ} \mathrm{C}$. In the third phase, all samples were soaked into sodium alginate solution $(6 \mathrm{~g} / \mathrm{l})$ for 30 seconds. They were identically passed through the foulard that was set at $100 \%$ uptake. The antimicrobial properties were generated by addition of silver nitrate solution $(0.05 \mathrm{~g} / \mathrm{l})$ [9]. At this point, half of the finished samples in the previous steps were used, and the other half went under antimicrobial finishing by chitosan solution $(0.05 \mathrm{~g} / \mathrm{l})$. These two phases were carried out under the same conditions. The samples finished with the chitosan solution did not go under finishing with sodium alginate. In the final stage, odor-absorbing finishing was performed using aluminum oxide solution $(0.025 \mathrm{~g} / \mathrm{l})$. All samples were padded and thermally fixed using the laboratory foulard and their specific amounts.

Tests: The standard method of ASTM D 4772$09 \mathrm{e} 1$ was used to measure water absorption rate. Water drop penetration time, dispersion and water retention volume were measured. $S$. aureus ATCC 6538 was used to evaluate the antimicrobial activity of samples using the quantitative method provided by the American Association of Textile Chemists and Colorists [(AATCC) test method 100-1993] (12-13). First, the samples (with diameter of $5 \mathrm{~cm}$ ) were sterilized in the autoclave at $121{ }^{\circ} \mathrm{C}$ for 15 minutes. The bacteria were cultured in 10 $\mathrm{ml}$ nutrient broth and then incubated at $37{ }^{\circ} \mathrm{C}$ for 18-24 hours. One $\mathrm{ml}$ of the incubated nutrient broth was transferred to a sterile nutrient agar plate, and the plate was placed in the Incubator. A few loops from the culture medium was transferred to a test tube containing $9 \mathrm{ml}$ of normal saline. Concentration of the tube was evaluated by measuring absorbance at $580 \mathrm{~nm}$ using spectrophotometer (Spectronic 700). One $\mathrm{ml}$ of solution with a concentration of 1000 $\mathrm{CFU} / \mathrm{ml}$ was transferred onto the fabrics and then to a $250 \mathrm{ml}$ sterile Erlenmeyer flask using forceps. After 24 hours of incubation, $100 \mathrm{ml}$ of sterile distilled water were added to the flask and mixed for one minute. One $\mathrm{ml}$ of the solution was transferred to a plate containing $25 \mathrm{ml}$ of nutrient agar. The plate was incubated at $37{ }^{\circ} \mathrm{C}$ for 24 hours. After performing these steps, number of live bacteria was calculated using colony counter, and bacteria reduction rate was calculated using the following equation:

Reduction rate $(\%)=[(\mathrm{A}-\mathrm{B}) / \mathrm{A}] \times 100$

$\mathrm{A}=$ number of bacterial colonies on untreated sample

$\mathrm{B}=$ number of bacterial colonies on treated sample

Human olfactory test was done in accordance with ISO12 standards at $22 \pm 3{ }^{\circ} \mathrm{C}$ and $65 \%$ relative humidity. Untreated and treated samples and $200 \mathrm{~g}$ of chopped onion were confined within a closed container for 24 hours. The samples were then removed from the container and placed separately in a test tube with cap. The samples were given one hour so that their headspace could reach equilibrium for the olfactory testing (14).

For capillary rise testing, samples with dimensions of $15 \times 2.5 \mathrm{~cm}^{2}$ (both raw and finished fabrics) were prepared and one side of the samples were dipped inside one centimeter of water. The amount of risen water inside the sample was measured at different times. Small amounts of disperse water-soluble blue dye was used for better detection (15). The samples were coded according to the stages performed (Table 1). The following stages were performed:

Finishing with calcium chloride solution

Addition of sodium alginate solution to samples

Finishing with silver nitrate solution Addition of chitosan solution to samples Addition of aluminum oxide solution to samples. 
Table 1- The list of samples tested in this study

\begin{tabular}{lc}
\hline \multicolumn{1}{c}{ Operations performed on samples } & $\begin{array}{c}\text { Sample } \\
\text { Code }\end{array}$ \\
\hline Raw nonwoven sample 1 with paper weight of $85 \mathrm{~g} / \mathrm{m}^{2}$ & $\mathrm{~A}$ \\
Raw nonwoven sample 2 with paper weight of $140 \mathrm{~g} / \mathrm{m}^{2}$ & $\mathrm{~B}$ \\
Raw nonwoven sample 3 with paper weight of $190 \mathrm{~g} / \mathrm{m}^{2}$ & $\mathrm{C}$ \\
Raw nonwoven sample 4 with paper weight of $240 \mathrm{~g} / \mathrm{m}^{2}$ & $\mathrm{D}$ \\
Sample 1 treated under stages $1-5$ and dried at ambient temperature & $A_{1}$ \\
Sample 2 treated under stages $1-5$ and dried at ambient temperature & $B_{1}$ \\
Sample 3 treated under stages $1-5$ and dried at ambient temperature & $C_{1}$ \\
Sample 4 treated under stages $1-5$ and dried at ambient temperature & $D_{1}$ \\
Sample 1 treated under stages $1-5$ and dried at $100{ }^{\circ} \mathrm{C}$ & $A_{2}$ \\
Sample 2 treated under stages $1-5$ and dried at $100{ }^{\circ} \mathrm{C}$ & $B_{2}$ \\
Sample 3 treated under stages $1-5$ and dried at $100{ }^{\circ} \mathrm{C}$ & $C_{2}$ \\
Sample 4 treated under stages $1-5$ and dried at $100{ }^{\circ} \mathrm{C}$ & $D_{2}$ \\
Sample 1 treated under stages $1,2,5$ and dried at ambient temperature & $A_{3}$ \\
Sample 2 treated under stages $1,2,5$ and dried at ambient temperature & $B_{3}$ \\
Sample 3 treated under stages $1,2,5$ and dried at ambient temperature & $C_{3}$ \\
Sample 4 treated under stages $1,2,5$ and dried at ambient temperature & $D_{3}$ \\
Sample 1 treated under stages $1,2,5$ and dried at $100{ }^{\circ} \mathrm{C}$ & $A_{4}$ \\
Sample 2 treated under stages $1,2,5$ and dried at $100{ }^{\circ} \mathrm{C}$ & $B_{4}$ \\
Sample 3 treated under stages $1,2,5$ and dried at $100{ }^{\circ} \mathrm{C}$ & $C_{4}$ \\
Sample 4 treated under stages $1,2,5$ and dried at $100{ }^{\circ} \mathrm{C}$ & $D_{4}$ \\
Sample 4 treated under stages $1,4,5$ and dried at ambient temperature & $D_{5}$ \\
Sample 4 treated under stages $1,4,5$ and dried at $100{ }^{\circ} \mathrm{C}$ & $D_{6}$ \\
\hline
\end{tabular}

Figure 1- Water retention volume in the raw and finished samples (in accordance with Table 1)

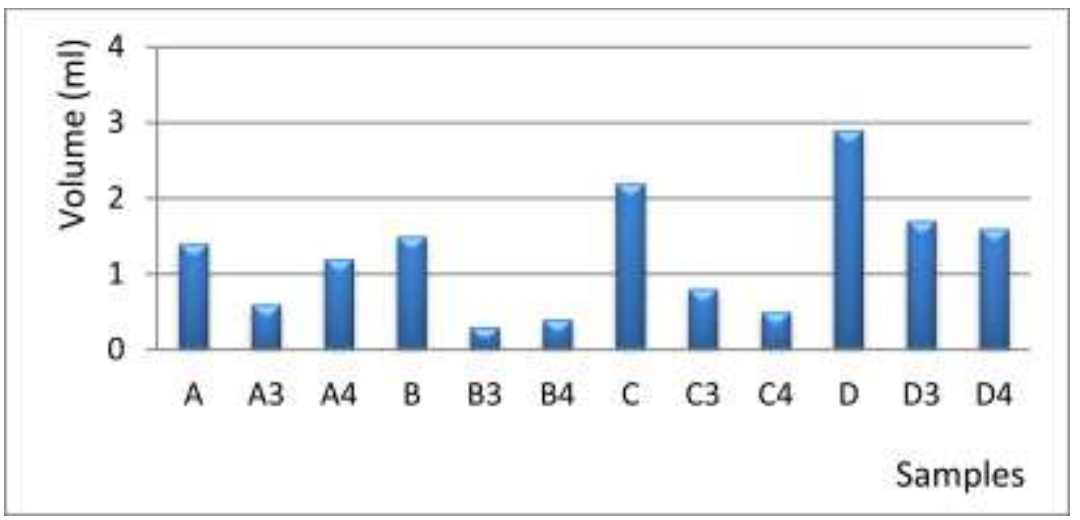

Figure 2-Water retention volume in the raw and chitosan-finished samples

D: raw sample, D5: sample finished with chitosan at ambient temperature, D6: sample finished with chitosan at $100{ }^{\circ} \mathrm{C}$

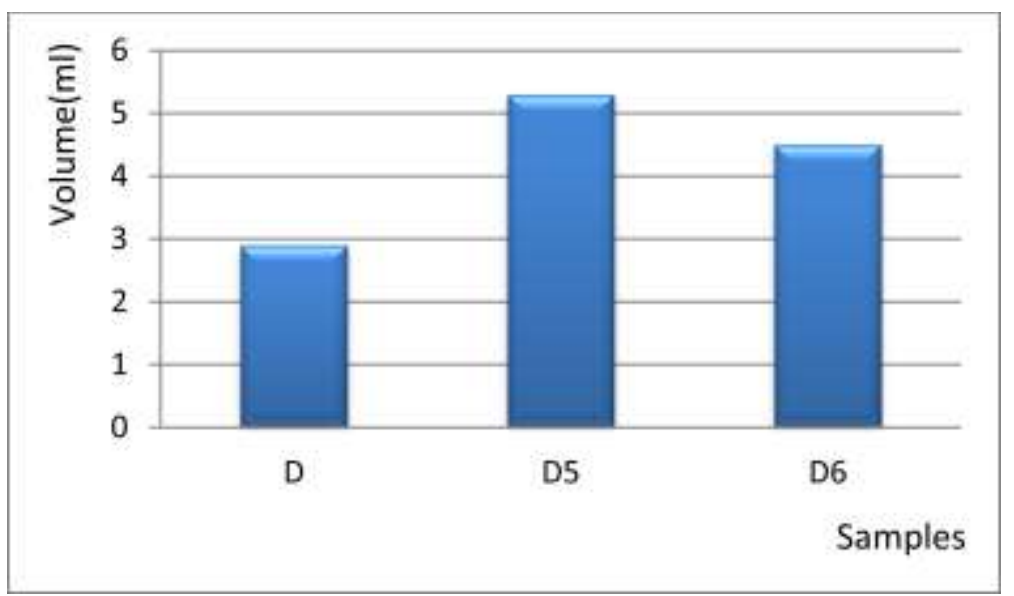




\section{RESULTS}

Water absorption test : After completing the finishing operations, the drop penetration time increased and the water retention volume decreased. Since there are many pores within the nonwoven sample, water can easily pass through the sample and no significant amount of water is retained. After finishing, the capillary flow rate of nonwoven fabric decreased because most of its pores were largely blocked by the finishing solutions. As a result, the finishing procedures did not improve hygroscopic properties. In addition, due to high concentration of sodium alginate, a film was formed on the fabric that improved the waterproof property. Before these samples reached saturation and prior to penetration to all levels, water droplets dripped from under the layer, and the nonwoven samples had nonuniform distribution. Addition of silver nitrate filled more pores, increased water drop penetration time and decreased the volume of water retained within the samples. Figure 1 shows the volume of water retained in the raw and finished samples.

Antibacterial testing: The antibacterial activity of samples against $S$. aureus is shown in Figure 3. In the samples treated with silver nitrate and chitosan, the significantly high percentage of bacteria reduction confirmed the antimicrobial properties of silver nitrate and chitosan.

Deodorization testing: Figures 4 and 5 show the results of deodorization testing of raw and finished samples. The results showed that the finished samples (according to Table 1) had lower odor intensity, and thus had better odorabsorbing ability. Deodorization of samples finished with chitosan can be attributed to aluminum oxide, and chitosan had no deodorizing effect (based on human olfaction, five and zero are the highest and lowest odor intensity odor).

Figure 3- Percentage of $S$. aureus reduction after culture on the finished samples D1: sample finished with silver nitrate at ambient temperature, D2: sample finished with silver nitrate at $100{ }^{\circ} \mathrm{C}$, D3: sample finished with chitosan at ambient temperature

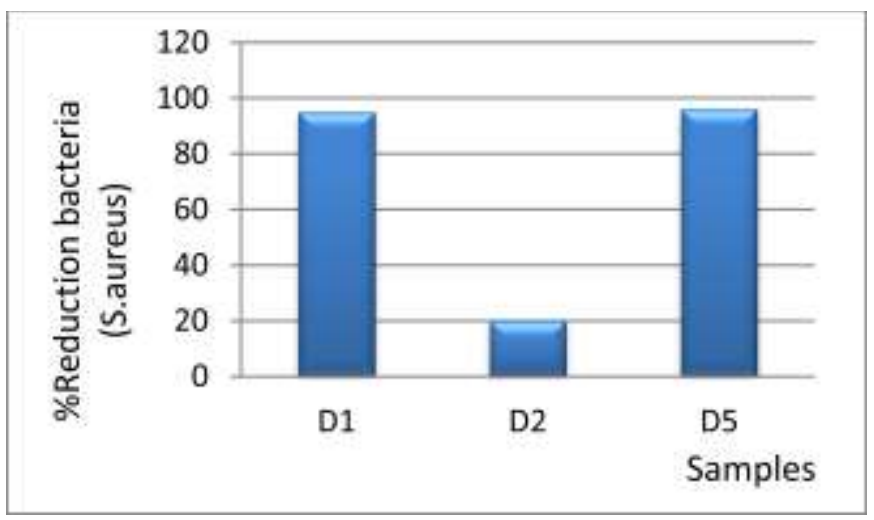

Figure 4- Deodorizing activity of raw and finished samples (the samples are presented in Table 1)

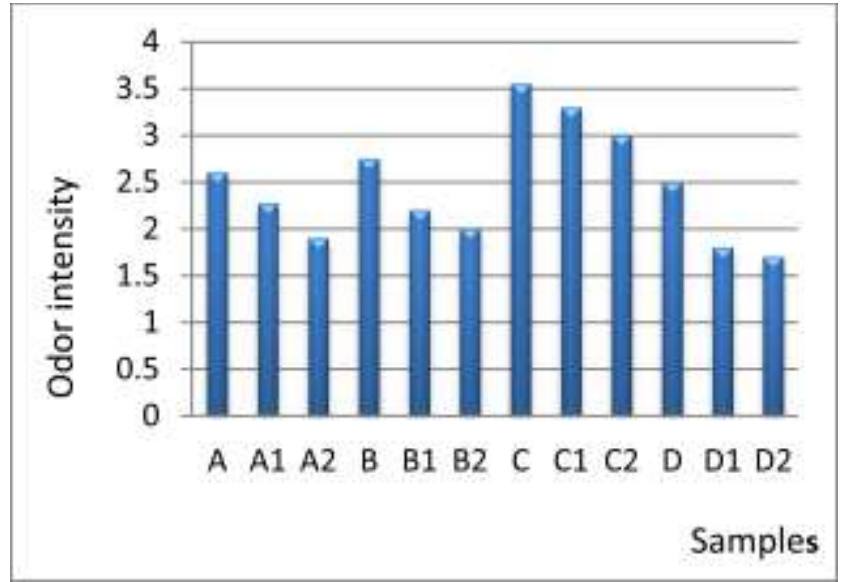


Capillary testing: Figure 6 shows the results of vertical wicking test for some of the samples. In this test, the level of water risen in different finished samples was measured at the same time. The wicking ability of samples decreased after finishing. This is because some of the capillary tubes in the fabric were filled with the solution used in the finishing process.

Figure 5- Deodorizing activity of raw and finished nonwoven samples (samples are presented in Table 1)

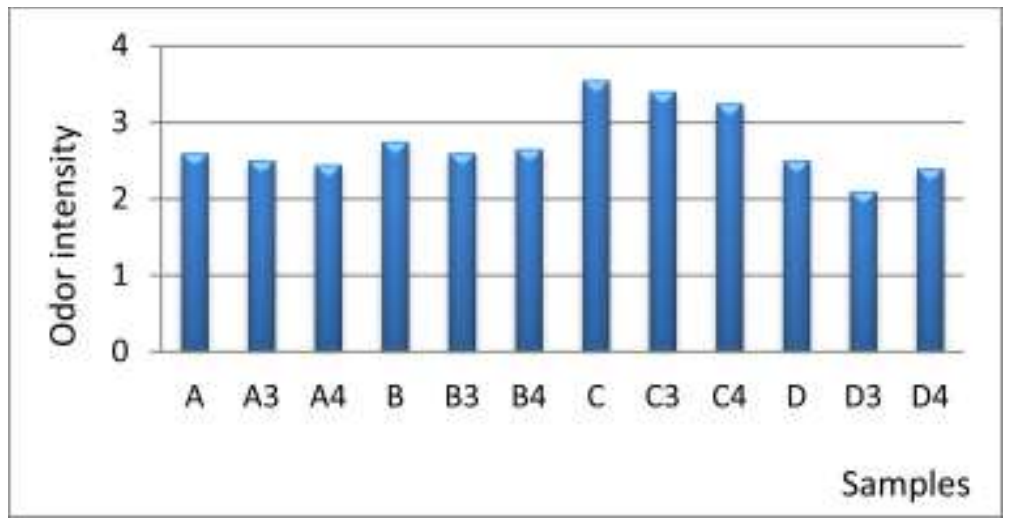

Figure 6- Results of capillary testing of raw and finished samples B

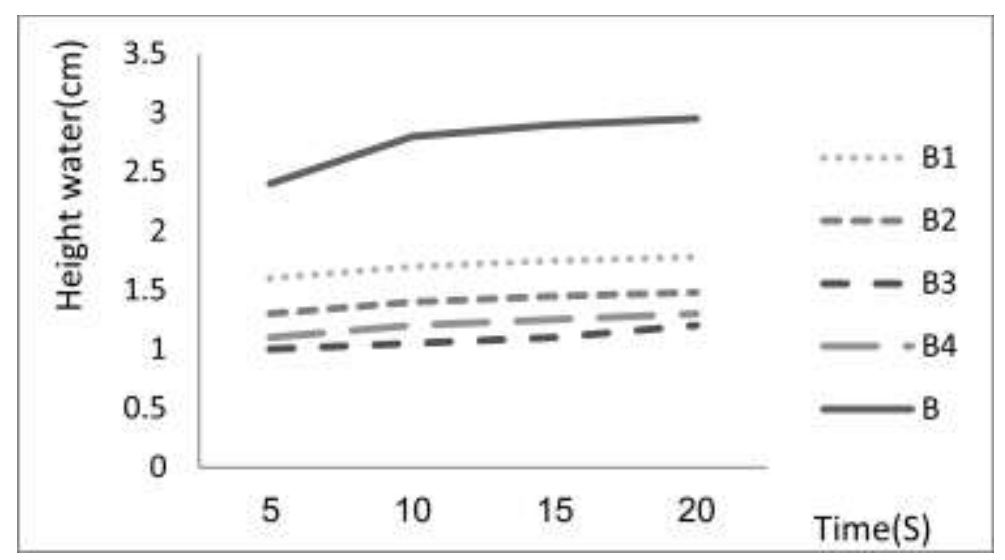

\section{DISCUSSION}

To the best of our knowledge, no similar study was found in scientific databases in this field. Given that medical textiles are among the growing important aspects of textile industry, this field has attracted a lot of attention. Recent technologies have successfully advanced in antimicrobial finishing of medical textiles. However, limited number of studies have been conducted to achieve simultaneous multiple processing on medical textiles (for example absorbing odors and wicking in addition to antimicrobial properties). The results showed that finishing with chitosan improved wicking ability and reduced water drop penetration time. It also increased the volume of water retained within the sample. The reason for this could be exclusion of two finishing phases and replacing them with one phase, which ultimately reduced water penetration and delayed saturation. Chitosan has a hydroxyl group, which is hydrophilic and absorbs water better. The antimicrobial activity of samples finished with silver nitrate at ambient temperature was significantly higher compared to samples finished at $100{ }^{\circ} \mathrm{C}$. This could be due to withdrawal of nanoparticles from the fabric at high temperatures. Samples finished with chitosan had the highest level of antimicrobial activity (about 98\%), which indicates higher antibacterial activity of chitosan in the products. Samples finished with silver nitrate had greater deodorizing activity compared to samples that 
were not finished with silver nitrate. This indicates that silver nitrate improved deodorization in finished samples. Capillary rise test results also indicated greater capillary activity reduction in samples with more weights. Moreover, the samples finished with chitosan instead of silver nitrate and sodium alginate, had lower capillary activity. This is because sodium alginate molecules are large, occupy a lot of space and makes difficult the possibility of water penetration.

\section{CONCLUSION}

This study used a simple, short and highly efficient method to improve water absorption, antimicrobial property and deodorization activity of non-woven polyester textile, using

\section{REFERENCES}

1. Dutta PK, Dutta J, Tripathi VS. Chitin and Chitosan: chemistry, properties and applications. J Scient Indust Res. 2004; 63(1): 20-31.

2. Lin S, Chen L, Huang L, Cao S, Luo X, Liu K. Novel antimicrobial chitosan-cellulose composite filmsbioconjugated with silver nanoparticles, Indus. Crop Prod. 2015; 70: 395-403.

3. Mathew T, Kuriakose S. Photochemical and antimicrobial properties of silver nanoparticleencapsulated chitosan functionalized with photoactive groups, Mater. Mater Sci Eng C Mater Biol Appl. 2013; 33(7): 4409-15. doi: 10.1016/j.msec.2013.06.037.

4. Lee S, Heo D, Moon J, Ko W, Lee J, Bae M, et al. Electrospun chitosan nanofibers with controlled levels of silver nanoparticles. Preparation, characterization and antibacterial activity. Carbohy. Polym. 2014; 111: 530537. doi:10.1016/j.carbpol.2014.04.026.

5. Gaafar MR, Mady RF, Diab RG, Shalaby ThI. Chitosan and silver nanoparticles: Promising antitoxoplasma agents. Exp Parasitol. 2014; 143: 30-8. doi: 10.1016/j.exppara.2014.05.005

6. Hebeisha AA, Ramadana MA, Montaser AS, Farag AM. Preparation, characterization and antibacterial activity of chitosan-g-poly acrylonitrile/silver nanocomposite. doi: 10.1016/j.ijbiomac.2014.04.028.

7. Mohamed RR, Saba MW. Synthesis and characterization of antimicrobial crosslinked carboxymethyl chitosan nanoparticles loaded with silver. Inter J Bio Macro. 2014; 69: 95-9. doi:10.1016/j.ijbiomac.2014.05.025.

8. Punitha N, Ramesh PS, Geetha D. Spectral, morphological and antibacterial studies of $\beta$ cyclodextrin stabilized silver-Chitosan nanocomposites. Spectrochim Acta A Mol Biomol Spectrosc. 2015; $136 \mathrm{Pt}$ C:1710-7. doi: 10.1016/j.saa.2014.10.071. biomaterials including chitosan, alginate, and nanoparticles of silver nitrate and aluminum oxide. The important and valuable benefits of the findings of this study could be useful for utilizing this procedure on other textiles such as sportswear and military textiles.

\section{ACKNOWLEDGEMENTS}

The authors would like to thank the supervisor of Research Laboratory of Yazd, Dr Hekmati Moghadam and personnel of the laboratory for carrying out the anti-microbial testing.

\section{CONFLICT OF INTEREST}

The authors declare no conflicts of interest regarding this manuscript.

9. Behary N, Kerkeni A, Perwuelz A, Chihib N, Dhulster P. Bioactivation of PET woven fabrics using alginate biopolymer and the bacteriocin nisin. Textile Res J. 2013; 83(11): 1120-29. doi: $10.1177 / 0040517512471743$.

10. Parikh D, Fink T, Rajasekharan K, Sachinvala N, Sawhney A, Calamari T. Antimicrobial Silver/Sodium Carboxymethyl Cotton Dressings for Burn Wounds. Textile Res J. 2005; 75(2): 134-8. doi: $10.1177 / 004051750507500208$

11. Selvam S, Rajiv Gandhi R, Suresh J, Gowri S, Ravikumar S, Sundrarajan M. Antibacterial effect of novel synthesized sulfated $\beta$-cyclodextrin crosslinked cotton fabric and its improved antibacterial activities with $\mathrm{ZnO}, \mathrm{TiO}_{2}$ and $\mathrm{Ag}$ nanoparticles coating. Int J Pharm. 2012; 434(1-2): 366-74. doi: 10.1016/j.ijpharm.2012.04.069.

12. Abedi D, Mortazavi SM, Khajeh Mehrizi M, Feiz M. Antimicrobial Properties of Acrylic Fabrics Dyed with Direct Dye and a Copper Salt. Textile Res J. 2008; 78(4): 311-9. doi: 10.1177/0040517508090486.

13. Khajeh Mehrizi M, Mortazavi SM, Abedi D. The Antimicrobial Characteristic Study of Acrylic Fiber Treated with Metal Salts and Direct Dyes. Fiber. Polym. 2009; 10(5): 601-5. doi:10.1007/s12221-010-0601-z.

14. Eza TSM, Ahmad WY Wan, Ahmad MR, Omar K, Ahmad MN. Effectiveness of activated carbon produced from coconut and oil palm shells as anti-odour on textile fabrics. Indian J Fib Tex Res. 2014; 39(2): 190-5.

15. Patnaik A, Rengasamy RS, Kothari VK, Ghosh A. Wetting an wicking in fibrous materials. Textile Prog. 2006; 38(1): 1-105. doi:10.1533/jotp.2006.38.1.1 